\title{
Behavioral Biases and Long Term Care Insurance: A Political Economy Approach ${ }^{1}$
}

\author{
Philippe De Donder ${ }^{2}$, Marie-Louise Leroux ${ }^{3}$
}

First version: October 2012. Revised version: February 2013

\footnotetext{
${ }^{1}$ A previous version of this paper has been presented at the 2012 Venice Summer Institute Workshop on "The Economics of Long-Term Care" under the title "A Political Economy Approach to Long-Term Care with Sophisticated or (In)Consistently Myopic Voters". We thank our discussant and three anonymous referees for their suggestions. Financial support from the Chaire "Marché des risques et création de valeur" of the FdR/SCOR is gratefully acknowledged. The usual caveat applies.

${ }^{2}$ Toulouse School of Economics (GREMAQ-CNRS and IDEI). Email: philippe.dedonder@tse-fr.eu

${ }^{3}$ Corresponding author. Département des Sciences Économiques, ESG - Université du Québec à Montréal. E-mail: leroux.marie-louise@uqam.ca
} 


\begin{abstract}
We develop a model where individuals all have the same probability of becoming dependent and vote over the social long term care insurance contribution rate before buying additional private insurance and saving. We study three types of behavioral biases, all having in common that agents under-weight their dependency probability when taking private decisions. Sophisticated procrastinators anticipate their mistake when voting, while optimistic and myopic agents have preferences that are consistent across choices. Optimists under-estimate their own probability of becoming dependent but know the average probability while myopics underestimate both. Sophisticated procrastinators attain the first-best allocation while myopics and optimists insure too little and save too much. Myopics and optimists more (resp., less) biased than the median are worse off (resp., better off), at the majority voting equilibrium, when private insurance is available than when it is not.

Keywords: Majority Voting, Myopia, Optimism, Sophisticated Procrastinators, Complementary Private Insurance, Dependency Linked Annuity.

JEL codes: H55, I13, D91.
\end{abstract}




\section{Introduction}

Long Term Care (LTC hereafter) "is the care for people needing support in many facets of living over a prolonged period of time. Typically, this refers to help with so-called activities of daily living, such as bathing, dressing, and getting in and out of bed" (OECD, 2011). People in need of LTC are called dependent.

LTC is an important public policy problem. According to Brown and Finkelstein (2009), in the US, between 35 and 50 percent of 65 year old will use a nursing home at some point in their remaining lives. Of those who use a nursing home, 10 to 20 percent will live there more than five years. The costs of formal LTC are also very large. Genworth (2012) surveys them for the US: in 2012 , the median annual rate for a private nursing room is more than $\$ 80,000$, while the median hourly rate charged for a home health aid is $\$ 19$. The issue of the financing of LTC needs will loom larger in the near future because of two trends: demographic (ageing of the population) and sociological (decrease in the amount of informal care provided by the family).

As argued by Brown and Finkelstein (2011, p122), "the possibility of needing long-term care is exactly the sort of large, uncertain expenditure risk for which insurance would seem to be most valuable. Yet most long-term care expenditure risk is not insured" in the US. A similar statement is made for OECD countries by OECD (2011). For instance, the share of (public and private) LTC expenditures accounted for $1.5 \%$ of GDP on average across 25 OECD countries in 2008, while the share of formal LTC expenditures covered by private LTC insurance varies from roughly $0.5 \%$ in France and Canada to a maximum of $5 \%$ to $7 \%$ in Japan and the United states. $^{1}$

The literature has offered several explanations for the lack of LTC insurance: ${ }^{2}$ asymmetric information problems (both moral hazard and adverse selection) induce insurers to restrict coverage (Sloan and Norton (1997)), informal help (mainly by family members) and social insurance crowd out the demand for private insurance (Brown and Finkelstein (2008)). Private insurance is afflicted by large loading factors and inefficiencies (Brown and Finkelstein (2007, 2009)) while social insurance faces its own set of inefficiencies plus the cost of public funds.

We focus here on a different reason for the low demand for LTC insurance: behavioral biases.

\footnotetext{
${ }^{1}$ Public LTC programs provide either cash benefits or in-kind services, which depend on the needs and on the financial resources of the user. Since benefits never totally cover the total cost of dependency, individuals have an incentive to complement public LTC coverage with private insurance. From now on, we use the term of "social insurance" for all such public programs. They include Medicaid in the US and the APA in France.

${ }^{2}$ For surveys on long term care, see Brown and Finkelstein (2011) and Cremer et al. (2009).
} 
Brown and Finkelstein (2009), Pestieau and Ponthière (2010) and Zhou-Richter et al. (2010) mention these biases as a primary reason for the lack of LTC insurance. The following quote from OECD (2011, p.253) shows that different phenomena may be at play: "For instance, the risk associated with dependency is often deemed as too remote to warrant coverage starting at a relatively young age. Individuals' perceptions on the level of public support also affect the perceived need to hold private coverage. These may translate in individuals delaying until an older age decisions regarding the purchase of a private LTC coverage, when they are more likely to face high premia and less likely to pass underwriting tests" (our emphasis). Biases may then have to do with the misperception of the future level of need, or may rather resemble procrastination, where individuals delay the decision to purchase insurance until it is too late, or at the very least until it gets very costly.

To the best of our knowledge, there is little literature trying to disentangle these different types of biases, and the first objective of our paper is to clarify the meaning of these behavioral biases by proposing different ways to model them. The second objective is then to assess the impact of these behavioral biases on the demand for both public and private LTC insurance, together with self-insurance (or saving).

To this end, we develop a simple two-period model where all individuals have the same income and the same probability of becoming dependent in the second period. In the first period, they first vote over an income tax rate whose proceeds are used to serve a lump-sum benefit in case of dependency. They then decide their preferred levels of saving and private LTC insurance. The behavioral biases that affect individuals translate into their putting too little weight on the probability of becoming dependent when saving and buying LTC insurance. Agents differ only according to how much they under-weight this future state of the world at this stage- i.e., by their degree of behavioral bias.

This under-weighting may come from a misperception problem, when individuals underestimate their own risk of becoming dependent. An extensive literature, covering different fields, shows that some individuals exhibit a so-called "Lake Wobegon effect, where all or nearly all of a group claim to be above average. (... It) has been observed among drivers, CEOs, hedge fund managers, presidents, coaches, radio show hosts, late night comedians, stock market analysts, college students, parents, and state education officials, among others." ${ }^{3}$ We call agents under-

\footnotetext{
${ }^{3}$ See wikipedia.org and the references mentioned there. See also the related Dunning-Kruger effect where agents mistakenly rate their ability much higher than average.
} 
estimating their own risk but assessing correctly the average, economy-wide, risk the optimists (or type $\mathrm{O}$ individuals).

Another type of misperception consists in under-estimating both one's own risk and the average risk of dependency in society. We call such individuals myopic (or type M). For them, dependency is simply seen as a smaller issue, in general, than what it is (or will be by the time they become old enough).

The little empirical literature that studies behavioral biases in LTC decisions tend to support this misperception problem. Finkelstein and McGarry (2006) compare the subjective probability of entering a nursing home within five years for respondents aged on average 78 to the actual decisions of the same respondents after five years. They find that most respondents underestimate their true probability of needing such a form of LTC. ${ }^{4}$ Also, based on a review of several surveys, Pauly (1990) attributes the non-purchase of private LTC insurance to the fact that individuals lack awareness of their probability of needing LTC services. This opinion is indirectly supported by a questionnaire conducted among financial planners by Bacon et al. (1989), in which the majority of respondents reported that their clients are unaware of their risk of a potential nursing home stay. At the same time, it is unclear from these studies whether individuals, who mostly under-estimate their own risk, also under-estimate the average risk. We then study both myopics and optimists in the rest of the paper, and we contrast their choices. It is worth stressing that both make the same mistake(s) when voting and when saving and buying private insurance: their preferences are consistent (and biased) across all choices.

Alternatively, the under-weighting of the probability of being dependent may arise from a procrastination problem: although they know perfectly their own (and the average) probability of becoming dependent in the future, individuals delay (as in the OECD quote above) the decision to buy LTC annuities. Observe that procrastination is much more difficult at the time of voting, since the election date is not under the control of agents. We then make the assumption that, at the time of voting, these individuals anticipate their future procrastination problem and thus use their true probability of becoming dependent. In other words, as in Cremer et al. (2007) and Haavio and Kotakorpi (2011), they vote "in a state of grace". We dub these individuals the

\footnotetext{
${ }^{4}$ To be fair, a non-negligeable fraction of respondents over-estimated their risk in this study. Also, comparing responses on subjective survival probabilities with actual ones, Hamermesh (1985) shows that middle aged individuals tend to under-estimate their survival probability to ages below 70 years old but over-estimate it for ages above 70. Ludwig and Zimper (2007) obtains similar results. Since the prevalence of dependency increases sharply with age, especially after 70, these misperceptions may lead to over-estimations of the dependency risk. We discuss in the Conclusion how over-estimation by some agents would affect our results.
} 
sophisticated procrastinators (or type $\mathrm{S}$ individuals). To the best of our knowledge, there are no empirical results available regarding the extent of procrastination for LTC decisions.

Our main results go as follows. Both type $\mathrm{M}$ and $\mathrm{O}$ end up with too little insurance at their most-preferred allocation, but for different reasons: myopics because they over-estimate their consumption if dependent (since they over-estimate the LTC insurance return by underestimating the fraction of agents who become dependent), and optimists because they consider insurance to be actuarially unfair (since they mistakenly consider that they constitute a betterthan-average risk). Also, very biased optimists prefer no insurance at all while very biased myopics always buy some insurance. This difference then constitutes a testable prediction of our model, which allows to disentangle optimists from myopics. We also obtain the less intuitive result that types $\mathrm{M}$ and $\mathrm{O}$ save too much at their most-preferred allocation. Consistent behavioral bias of types $\mathrm{M}$ and $\mathrm{O}$ thus not only decreases LTC insurance below socially optimal levels, but also unbalances the mix between saving and insurance in providing for old age.

Since there is little LTC insurance bought in practice, we also investigate whether the availability or not of private insurance impacts the demand for social and self-insurance. We obtain that it does not affect the most-preferred allocation (since private and social insurance are seen as perfect substitutes) but impacts the majority voting equilibrium. When private insurance is available, both $\mathrm{M}$ and $\mathrm{O}$ agents vote for no social insurance, because they all prefer to buy their most-preferred amount of insurance on the private market rather than being forced to consume too much (social) insurance. When private insurance is not available, the majority chosen amount of (social) insurance is determined by the agent with the median bias. We obtain that making private insurance unavailable is beneficial to the ( $\mathrm{M}$ and $\mathrm{O})$ individuals who are more biased than the median (and are forced to under-insure less than they would like and induced to reduce their over-saving), at the expense of those less biased than the median (who are forced to insure even less than they would wish, and over-save more).

In stark contrast, type S agents make use of social insurance to obtain the socially optimal amount of insurance. This in turn neutralizes their procrastination when choosing how much to save. This result holds true both at their most-preferred allocation and at the majority chosen one, and whether private insurance is available or not (since no type S buys any private insurance). This does not fit the stylized facts, as there is little (social) LTC insurance in practice. 
We now provide a brief overview of the related literature. Our paper is linked to two emerging strands of the political economy literature. ${ }^{5}$ The first one consists of political economy models of LTC. Nuscheler and Roeder (2010)'s aim is to explain income redistribution and public financing of LTC, in a setting where voters differ in income and in LTC needs and where a proportional income tax finances both a lump-sum and a LTC transfer. De Donder and Pestieau (2011) studies the determinants of the demand for public and private LTC insurance, when individuals differ in income, in risk and in access to informal care. The second strand of political economy literature deals with agents having self-control problems. Cremer et al. (2007) models an economy composed of both fully rational individuals and fully sophisticated procrastinators, who also differ in income, and who vote over the size and the degree of income redistribution of the pay-as-you-go pension system. Haavio and Kotakorpi (2011) compares the optimal level of sin tax with the level chosen at the majority when individuals have different degrees of selfcontrol problems. As in Cremer et al. (2007), they assume that agents are sophisticated when voting, but they allow for a continuous distribution of the degree of behavioral bias.

To the best of our knowledge, our paper is then the first to propose this distinction between myopics and optimists and to provide a political economy analysis with both different types and different degrees of behavioral biases.

The paper is organized as follows. We describe the model and individuals' preferences in section 2 . We study the socially optimal allocation in section 3 . We then solve the model by backward induction, starting with the choice of private insurance and saving in section 4 . We study the amount of social LTC insurance chosen at equilibrium, assuming that all individuals are either myopic (section 5), optimistic (section 6) or sophisticated procrastinators (section 7). We discuss our results and conclude in section 8.

\section{The model}

We develop a two-period model with a mass one of individuals. In the first period, agents are young and all earn the same income $w$. They choose how much to save and insure for future LTC needs. In the second period, individuals are old and face the same probability $\pi$ to be

\footnotetext{
${ }^{5}$ Cremer and Roeder (2012) takes a normative perspective and examines whether misperception of individual LTC risks and private insurance market loading costs can justify social LTC insurance and/or subsidization of private insurance. Although they use the term of myopia, their agents are indeed optimistic since they correctly estimate the average dependency rate in the economy. They differ in their degree of bias, income and dependency risk, with the latter two positively and perfectly correlated.
} 
dependent and $(1-\pi)$ to remain autonomous. They take no decisions when old. We assume w.l.o.g. that the gross rate of interest is one.

Young individuals pay an income tax at a proportional rate $\tau$ whose proceeds finance a social LTC program serving a lump-sum benefit $b$ in case of dependency. The budget balance equation of this program depends crucially on the average probability of becoming dependent (which, by the law of large numbers, equals the proportion of elderly who become dependent). Because of the behavioral biases (detailed at greater length later) exhibited by the agents, it is important to distinguish the correct average dependency rate, $\pi$, from the perceived one, which we denote by $\bar{\pi}$. Once they have incorporated the government's budget constraint, individuals perceive that they will receive the benefit

$$
b(\bar{\pi})=\frac{\tau w}{\bar{\pi}},
$$

in case of dependency, while the correct level of the transfer is $b(\pi)$.

In the first period of their life, individuals decide how much to save and how much private insurance to buy. We assume that private insurance is available through a LTC annuity (called a Disability Linked Annuity, or DLA), which is a private financial product yielding a return differentiated upon the health condition of the individual. ${ }^{6}$ The perceived return of this annuity is $R^{h}(\gamma)$ in case of autonomy, with

$$
R^{h}(\gamma)=1-\gamma
$$

and $R^{d}(\gamma, \bar{\pi})$ in case of dependency, with

$$
R^{d}(\gamma, \bar{\pi})=1-\gamma+\frac{\gamma}{\bar{\pi}}=1+\gamma \frac{1-\bar{\pi}}{\bar{\pi}} \geq R^{h} .
$$

The exogenous parameter $\gamma \in[0,1]$ represents the fraction of the annuity made of insurance while the remaining part is the fraction devoted to saving. When $\gamma=0$, we have pure saving since the return from investing in the private annuity is independent of the LTC condition of the agent and equals the gross interest rate of one. When $\gamma=1$, we have pure insurance since the individual gets nothing if autonomous but believes he obtains a gross return of $1 / \bar{\pi}>1$ if dependent. Raising $\gamma$ thus increases the return in case of dependency at the expense of the

\footnotetext{
${ }^{6}$ See Mayhew et al. (2010) and Rickayzen (2007). It is also called an enhanced life annuity (Levantesi and Menzietti (2012)). This product is currently available in the US. We model DLAs for two reasons. First, the instrument is interesting by itself: OECD (2011) considers that it is one of "the most promising private sector innovations", while Mayhew et al. (2010) states that "DLAs could make an important contribution to LTC planning." Second, as shown below, by varying $\gamma$ we can cover the case where private insurance is available $(\gamma>0)$ and where it is not $(\gamma=0)$.
} 
return when autonomous. Finally, the perceived return when dependent is decreasing in the perceived average prevalence of dependency, $\bar{\pi}$, when $\gamma>0$.

Since there is little LTC insurance bought in practice, our approach allows both to sudy how we can rationalize this lack of demand, and also to ascertain whether the availability of private insurance does impact the demand for social and self-insurance.

An agent's true (or correct) utility function is given by:

$$
\begin{aligned}
& u(c)+(1-\pi) u\left(d^{h}\right)+\pi v\left(d^{d}(\pi)\right) \\
& \text { with }\left\{\begin{array}{l}
c=w(1-\tau)-a-s \\
d^{h}=s+R^{h}(\gamma) a \\
d^{d}(\pi)=s+R^{d}(\gamma, \pi) a+b(\pi)
\end{array}\right.
\end{aligned}
$$

where $c$ is first-period consumption, $d^{h}$ and $d^{d}(\pi)$ are second-period consumptions in case of, respectively, autonomy and dependency, $s$ is saving and $a$ denotes the amount of LTC annuity.

We assume that both the utility function in case of autonomy, $u($.$) , and in case of depen-$ dency, $v($.$) , are increasing and concave \left(u^{\prime}>0, u^{\prime \prime}<0, v^{\prime}>0\right.$ and $\left.v^{\prime \prime}<0\right)$ and that $u^{\prime}(0)=\infty$. Moreover, we assume that

$$
v(x)=u(x-L),
$$

i.e., becoming dependent is equivalent to suffering a monetary loss $L>0 .^{7}$

As for the sequence of decisions, we assume that young individuals vote first over the value of the social LTC insurance contribution rate, $\tau$, that they observe the majority chosen level, and that they then privately decide the amount of private LTC annuity that they wish to buy, $a$ and the amount of savings, $s$ they want to make. ${ }^{8}$

We consider and contrast three distinct forms of behavioral biases. All have in common that they under-estimate their probability of becoming dependent by a factor $\alpha$ when choosing $a$ and

\footnotetext{
${ }^{7}$ This assumption allows us to obtain explicit formulations for the optimal allocation (section 3 ) and the one most-preferred by myopic agents (section 5). Our results would hold qualitatively more generally provided that $u(x)>v(x)$ and $u^{\prime}(x)<v^{\prime}(x), \forall x$. These assumptions reflect the observations that, for any consumption level, individuals are worse off if dependent than if autonomous, but have higher needs (i.e. higher marginal utility from consumption). The latter assumption may be disputed (see for instance Finkelstein et al., 2013) since some goods may substitute or complement good health. Observe that if dependent people do not have higher marginal utility than when autonomous, the lack of demand for LTC insurance is not puzzling at all.

${ }^{8}$ We assume that the transfer received by agents when old is determined by the contribution rate $\tau$ implemented when they were young. Since any change in the current contribution rate only affects the future payments received by the currently young agents, agents vote only when young. Alternatively, we could have studied an overlappinggeneration model with a pay-as-you-go LTC insurance, where variations in $\tau$ impact both the (currently) young and old agents. In that case, old agents would either be indifferent as to the value of $\tau$ (if autonomous at the time of voting), or favor the maximum value of $\tau$ (if dependent). As for young agents, their preference would depend on the perceived link between current contribution and future transfer. Such considerations are well known in the political economy literature on pensions (see Sjoblom, 1985), and we abstract from them here.
} 
$s$. More precisely, they all consider their probability to become dependent as $\alpha \pi$ rather than $\pi$. Agents differ according to this bias parameter $\alpha$, which is distributed in the economy on the interval ]0,1] with a median value denoted by $\alpha_{\text {med }}{ }^{9}$

Sophisticated procrastinators anticipate this mistake when voting over the social insurance tax rate: they use the correct probability $\pi$ of being dependent when voting over $\tau$, anticipating that they will use biased probabilities $\alpha \pi$ when deciding later over $a$ and $s$. With this interpretation, there is no reason for $\mathrm{S}$ individuals to mis-estimate the aggregate probability of being dependent, so that they use $\bar{\pi}=\pi$ in their calculation of both private and public insurance returns (see first set of rows of Table 1).

\begin{tabular}{|l|l|c|c|}
\hline Type & Choice & Weight on $d$ state & Perceived average risk $\bar{\pi}$ \\
\hline \hline \multirow{2}{*}{$\mathrm{S}$} & vote over $\tau$ & $\pi$ & $\pi$ \\
\cline { 2 - 4 } & choice of $a / s$ & $\alpha \pi$ & $\pi$ \\
\hline \hline \multirow{2}{*}{$\mathrm{O}$} & vote over $\tau$ & $\alpha \pi$ & $\pi$ \\
\cline { 2 - 4 } & choice of $a / s$ & $\alpha \pi$ & $\pi$ \\
\hline \hline \multirow{2}{*}{$\mathrm{M}$} & vote over $\tau$ & $\alpha \pi$ & $\alpha \pi$ \\
\cline { 2 - 4 } & choice of $a / s$ & $\alpha \pi$ & $\alpha \pi$ \\
\hline
\end{tabular}

Table 1: Parameters used by individuals in both decisions

Unlike sophisticated procrastinators, the other two types of behavioral biases exhibit consistent preferences when voting over $\tau$ and choosing $a$ and $s$. The optimists anticipate correctly the average probability of dependency $\pi$ but under-estimate their own risk as $\alpha \pi$ (see second set of rows of Table 1). By contrast, myopic agents similarly under-estimate both their individual and the average probability of becoming dependent. Since myopics under-estimate the future prevalence of LTC for all individuals in the economy, and do not consider themselves to be different from others, they use $\alpha \pi$ as both their individual and average probability (see last set of rows of Table 1). Type $\mathrm{M}$ is the only type making a mistake when anticipating the return of both social insurance and the LTC annuity (at least as long as it embodies some insurance characteristics-i.e., when $\gamma>0$ ).

Finally, we assume that society is homogenous in the sense that it consists exclusively of agents of either type $\mathrm{S}$, type $\mathrm{M}$ or type $\mathrm{O}$. We explain in section 8 how our results would be affected if they were to coexist in the same society.

\footnotetext{
${ }^{9}$ In the rest of the paper, we assume that no agent is totally myopic, that is to say, they all believe that they have at least a very small chance of becoming dependent: $\alpha>0$ for all. This is a reasonable assumption that allows to simplify our analysis since we can disregard any discontinuity in saving or voting behavior when $\alpha=0$. In the conclusion, we discuss how our results would be affected if some agents over-estimated their risk, $\alpha>1$.
} 
We start by identifying the socially optimal allocation that will be used as a benchmark to assess the majority-voting equilibria studied in subsequent sections of the paper.

\section{The socially optimal allocation}

Whatever the type of behavioral bias, agents make a mistake when assessing how much saving and DLA to buy. Some of them anticipate this mistake (the sophisticated procrastinators) while others regret their past decisions once they become dependent (the myopics, whose consumption level is lower than anticipated). Whether they realize or anticipate their mistake or not, there is a case to be made for a paternalistic approach-i.e., to evaluate the allocations using the correct (or true) preferences of the agents, rather than the one that they mistakenly use. ${ }^{10}$ The socially optimal allocation then corresponds to the allocation $\left(c, d^{h}, d^{d}\right)$ that maximizes $(4)$. It corresponds to the allocation chosen by an individual without behavioral bias (i.e., with $\alpha=1$ ). It is easy to show that the optimal allocation is such that marginal utilities are equalized across states of nature:

$$
u^{\prime}(c)=u^{\prime}\left(d^{h}\right)=v^{\prime}\left(d^{d}\right) .
$$

Equalizing marginal utilities across periods and states of the world requires two types of transfers. To see this, we denote by $S$ the amount of total saving (i.e., second-period transfer enjoyed whether dependent or not) and by $I$ the total amount of (public and private) insurance (i.e., transfer received only if dependent):

$$
\begin{aligned}
S & \equiv(1-\gamma) a+s \\
I & \equiv \frac{\tau w+\gamma a}{\pi} .
\end{aligned}
$$

Total saving corresponds to the share $1-\gamma$ of the LTC annuity, plus private savings $s$, while total insurance is made of both social insurance (financed with $\tau$ ) and of the share $\gamma$ of the LTC annuity, with the total "premium" $\tau w+\gamma a$ sharing the same return, $1 / \pi$.

The correct utility of an individual, as given by (4), can then be reformulated as

$$
W(I, S)=u(w-S-\pi I)+(1-\pi) u(S)+\pi v(S+I) .
$$

\footnotetext{
${ }^{10}$ See O'Donoghue and Rabin (2003) and Thaler and Sunstein (2003) for a discussion of the "new paternalism" approach linked to the rise of behavioral economics. See Salanie and Treich (2009) for a paternalistic social planner in a context where agents misperceive risks.
} 
Denoting the optimal values of variables by the superscript opt, we then obtain that

$$
\begin{aligned}
S^{o p t} & =\frac{w-\pi L}{2}, \\
I^{\text {opt }} & =L .
\end{aligned}
$$

The socially optimal amount of insurance corresponds to the LTC loss, $L$, equalizing marginal utilities in the two states of the world in the second period. The optimal saving amount equalizes marginal utility across periods. In the rest of the paper, we assume that the socially optimal allocation is feasible- i.e., that $w>\pi L$.

The decentralization of (5) and (6) depends on the value of $\gamma$. When $\gamma=0$, DLAs are equivalent to saving so that $a$ and $s$ are perfect substitutes while (6) determines a unique value of $\tau$. When $\gamma=1$, DLAs are equivalent to private insurance so that $\tau$ and $a$ are perfect substitutes and (5) determines a unique value of $s$. Finally, when $0<\gamma<1$, there is a continuum of values of $a, s$ and $\tau$ that satisfy (5) and (6), since the DLA is a mix of saving and insurance.

We now turn to the study of the equilibrium with behavioral biases. We start with the second stage choice of saving $s$ and DLA $a$, and then move to the choice of the social insurance rate $\tau$.

\section{Choice of saving and DLA}

The agents perceived preferences when choosing $a$ and $s$ are given by

$$
\begin{aligned}
U= & u(w(1-\tau)-a-s) \\
& +(1-\alpha \pi) u\left(R^{h}(\gamma) a+s\right)+\alpha \pi v\left(b(\bar{\pi})+R^{d}(\gamma, \bar{\pi}) a+s\right),
\end{aligned}
$$

with the value of $\tau$ exogenous at this stage. Both public and private insurance transfers are obtained using the return perceived by the individual, as given by (1), (2) and (3) respectively.

First-order conditions with respect to $s$ and $a$ are:

$$
\begin{aligned}
& \frac{\partial U}{\partial s}=-u^{\prime}(c)+(1-\alpha \pi) u^{\prime}\left(d^{h}\right)+\alpha \pi v^{\prime}\left(d^{d}(\bar{\pi})\right) \leq 0 \\
& \frac{\partial U}{\partial a}=-u^{\prime}(c)+(1-\alpha \pi) R^{h}(\gamma) u^{\prime}\left(d^{h}\right)+\alpha \pi R^{d}(\gamma, \bar{\pi}) v^{\prime}\left(d^{d}(\bar{\pi})\right) \leq 0
\end{aligned}
$$

where $d^{d}(\bar{\pi})$ is the perceived future consumption of the individual in case of dependency. Intuitively, the individuals plan to equalize the marginal cost and benefit of saving (equation (8)) and of buying DLAs (equation (9)), with the latter reflecting the probability of each future state 
of the world, and the state-dependent marginal utility. Behavioral biases translate into too large a weight being given to the autonomous state (for all types of agents), and, for myopics only, into a mis-perception of the consumption level in case of dependency (because of over-estimation of the public and private insurance transfers). The two FOCs differ in the expected return of the two instruments.

We denote by $s^{*}(\alpha, \gamma, \tau, \bar{\pi})$ and $a^{*}(\alpha, \gamma, \tau, \bar{\pi})$, respectively, the agent's most-preferred saving and DLA amounts in this second stage. Applying Cramer's rule on (8) and (9), we find that when $\gamma>0$,

$$
\frac{d s^{*}(\alpha, \gamma, \tau, \bar{\pi})}{d \tau}>0 \text { and } \frac{d a^{*}(\alpha, \gamma, \tau, \bar{\pi})}{d \tau}<0 .
$$

There is substitution between social and private insurance, so that agents buy less DLAs when $\tau$ increases, but compensate the induced decrease in total saving by increasing $s^{*}$.

When $\gamma=0$, DLAs are equivalent to saving, so that the two FOCs become identical and simplify to

$$
u^{\prime}(c)=(1-\alpha \pi) u^{\prime}(s)+\alpha \pi v^{\prime}(b(\bar{\pi})+s) .
$$

We assume w.l.o.g. that $a^{*}=0$ and use the implicit function theorem on (10) to obtain

$$
\frac{d s^{*}(\alpha, 0, \tau, \bar{\pi})}{d \tau}<0
$$

Unlike what happens when $\gamma>0$, there is substitutability between private savings $s$ and social insurance $\tau$ at the equilibrium: a larger social LTC insurance program raises the marginal (firstperiod) utility cost of saving and decreases its expected marginal (second-period) utility benefit, leading to a lower level of saving.

We proceed to the choice by majority voting of the social LTC insurance level. We start by studying the case where all individuals are myopic (section 5) before moving to the cases where they are optimistic (section 6) and then sophisticated (section 7).

\section{$5 \quad$ The myopics}

The (perceived) indirect utility function of myopics (who similarly under-estimate average and individual LTC risks) at the time of voting is given by

$$
\begin{aligned}
V_{M}(\alpha, \gamma, \tau)= & u\left(w(1-\tau)-s_{M}^{*}(\alpha, \gamma, \tau)-a_{M}^{*}(\alpha, \gamma, \tau)\right) \\
& +(1-\alpha \pi) u\left(R^{h}(\gamma) a_{M}^{*}(\alpha, \gamma, \tau)+s_{M}^{*}(\alpha, \gamma, \tau)\right) \\
& +\alpha \pi v\left(\tilde{b}+\tilde{R}^{d} a_{M}^{*}(\alpha, \gamma, \tau)+s_{M}^{*}(\alpha, \gamma, \tau)\right),
\end{aligned}
$$


where $s_{M}^{*}(\alpha, \gamma, \tau)=s^{*}(\alpha, \gamma, \tau, \alpha \pi), a_{M}^{*}(\alpha, \gamma, \tau)=a^{*}(\alpha, \gamma, \tau, \alpha \pi), \tilde{b}=b(\alpha \pi)>b(\pi)$ and $\tilde{R}^{d}=$ $R^{d}(\gamma, \alpha \pi)>R^{d}(\gamma, \pi)$ when $\gamma>0$.

Differentiating $V_{M}$ with respect to $\tau$, and making use of the envelope theorem for the optimal choices of saving and DLA, we obtain the following FOC

$$
\frac{\partial V_{M}(\alpha, \gamma, \tau)}{\partial \tau}=w\left[v^{\prime}\left(\tilde{d}^{d}\right)-u^{\prime}(c)\right]=0,
$$

where $\tilde{d}^{d}=d^{d}(\alpha \pi)>d^{d}(\pi)$. The intuition for this result is that $\mathrm{M}$ individuals consider the social LTC insurance as actuarially fair, since it is based on the same (mis-perceived) average risk as the individual risk, $\alpha \pi$. They use social insurance to equalize perceived marginal utilities when young and when dependent. Observe that the only way myopia intervenes in this formula is through $\tilde{d}^{d}$ : M individuals do not weight their marginal utility if dependent by $\alpha$, because the under-estimation of the average risk neutralizes the under-estimation of their own risk. Observe also that (11) holds whatever the value of $\gamma$.

Replacing for $v^{\prime}\left(\tilde{d}^{d}\right)=u^{\prime}(c)$ in (8) and (9), we obtain that the most-preferred allocation of myopic agents satisfies (for any $\gamma$ and any $\alpha>0$ )

$$
u^{\prime}(c)=u^{\prime}\left(d^{h}\right)=v^{\prime}\left(\tilde{d}^{d}\right)<v^{\prime}\left(d^{d}\right) .
$$

Myopics then aim at equalizing the marginal utilities in the three states of the world, but they over-estimate their consumption level when dependent.

We now look at the combination of insurance and saving that $M$ agents most prefer. Since myopic agents have the same preferences when choosing $\tau, s$ and $a$, their preferences can be reformulated in terms of the amounts of saving, $S$, and insurance transfer $\tilde{I}$ that they think they will obtain in the second period:

$$
u(w-S-\alpha \pi \tilde{I})+(1-\alpha \pi) u(S)+\alpha \pi v(S+\tilde{I}) .
$$

It is well known that risk averse agents fully insure themselves when offered an actuarially fair insurance contract (see Mossin (1968)) and this is what myopics think they are doing, by setting at equilibrium

$$
\tilde{I}_{M}^{*} \equiv \frac{\tau w+\gamma a}{\alpha \pi}=L
$$

This amount does not depend on $\alpha$ nor on $\gamma$. Observe that the actual amount of insurance payment received at the most-preferred allocation, $I_{M}^{*}$, is lower than optimal, since

$$
I_{M}^{*}(\alpha)=\frac{\tau w+\gamma a}{\pi}=\alpha \tilde{I}_{M}^{*}=\alpha L
$$


and increases with $\alpha$ : although the targeted amount of insurance $\tilde{I}_{M}^{*}$ does not change with $\alpha$, agents with a larger value of $\alpha$ under-estimate less the insurance return and end up with a larger insurance transfer.

The most-preferred total saving amount is given by

$$
S_{M}^{*}(\alpha)=s+(1-\gamma) a=\frac{w-\alpha \pi L}{2},
$$

so that $\mathrm{M}$ agents actually save too much compared to the social optimum, and that over-saving decreases continuously to zero as agents become less myopic (i.e., as $\alpha$ tends toward one). Looking back at condition (12), we see that they end up with too little consumption in the case they become dependent: optimality would require larger payments for insurance in the first period, which would increase marginal utility in the first period and thus decrease the amount of saving purchased compared to (15).

In order to assess normatively the equilibrium allocation chosen by individuals with behavioral biases, we use the correct utility function of agents (equation (7)), but measured at the allocation chosen by the myopic individual. ${ }^{11}$ The real utility attained at his most-preferred allocation by a myopic agent is then

$$
W_{M}^{*}(\alpha)=W\left(I_{M}^{*}(\alpha), S_{M}^{*}(\alpha)\right)
$$

This utility level is not affected by the value of $\gamma$ (and whether private insurance is available or not) but increases with $\alpha$, since both the choices of $I_{M}^{*}(\alpha), S_{M}^{*}(\alpha)$ and the real utility level converge to their socially optimal levels as $\alpha$ tends toward one.

We now move to the choice of social insurance by majority voting, and we show that the availability of private insurance does impact the equilibrium (as opposed to most-preferred) allocation.

When $\gamma>0$, social insurance can be complemented on the private market by buying DLAs. Myopic agents are then indifferent between any values of $\tau$ that give them no more social insurance than the optimal amount of total insurance they would like to consume (as given by (13)) -i.e. between any values of $\tau$ lower than (or at most equal to) $\hat{\tau}(\alpha)=\alpha L \pi / w$. Since they can not buy negative amounts of private insurance, their perceived utility is then decreasing in $\tau$ above this threshold $\hat{\tau}(\alpha)$. The unique majority-voting equilibrium (or Condorcet winning)

\footnotetext{
${ }^{11}$ For the justification of this approach, see the first paragraph of section 3.
} 
value of $\tau$, denoted by $\tau_{M}^{V}(\gamma)$, is then zero, since any positive value of $\tau$, say $\tau^{\circ}$, is defeated with the support of myopic agents with $\hat{\tau}(\alpha)<\tau^{\circ}$ (other agents are indifferent and we assume that they either abstain or throw a fair coin when deciding which tax rate to support).

By contrast, when $\gamma=0$ the only way to obtain insurance is to support a positive value of $\tau$. We denote by $\tau_{M}^{*}(\alpha, 0)$ the most-preferred tax rate of myopic individuals when $\gamma=0$, and solving the first-order condition (14), we obtain that

$$
\tau_{M}^{*}(\alpha, 0)=\frac{\alpha \pi L}{w} .
$$

Since preferences over $\tau$ are single-peaked (indeed, concave), there exists a unique majority winning value of $\tau$, which is the one most-favored by the agent with the median value of $\alpha$, $\alpha_{m e d}:$

$$
\tau_{M}^{V}(0)=\tau_{M}^{*}\left(\alpha_{m e d}, 0\right)=\frac{\alpha_{m e d} \pi L}{w}
$$

In the rest of this section, we compare the real utility of agents at the majority voting equilibrium according to whether private insurance is available $(\gamma>0)$ or not $(\gamma=0)$. We denote by $W_{M}^{V}(\alpha, \gamma)$ this real utility level. When $\gamma>0$, we have that

$$
W_{M}^{V}(\alpha, \gamma)=W_{M}^{*}(\alpha)
$$

while, when $\gamma=0$, we have ${ }^{12}$

$$
W_{M}^{V}(\alpha, 0)=W\left(\alpha_{\text {med }} L, s_{M}^{*}\left(\alpha, 0, \tau_{M}^{*}\left(\alpha_{m e d}, 0\right)\right)\right)
$$

In words, with $\gamma>0$, we have $\tau_{M}^{V}(\gamma)=0$ so that all agents obtain at the majority voting equilibrium the same utility level as at their most-preferred allocation (since they are indifferent between private and social insurance and thus buy their most-preferred insurance level on the private market). With $\gamma=0$, the total insurance amount is set at the majority chosen social insurance level $I_{M}^{*}\left(\alpha_{m e d}\right)$ and agents then choose their most-preferred saving level.

We claim that very myopic agents are actually better off, at the majority voting equilibrium, when private insurance is not available-i.e., that $W_{M}^{V}(\alpha, 0)>W_{M}^{V}(\alpha, \gamma)$ for any $\gamma>0$ and $\alpha<\alpha_{\text {med }}$. When left to their own devices, myopics save too much and buy too little insurance. In the absence of private insurance $(\gamma=0)$, very myopic individuals $\left(\alpha<\alpha_{\text {med }}\right)$ are forced to consume more insurance than they would wish (although less than what would be socially

\footnotetext{
${ }^{12}$ We assume w.l.o.g. that $a_{M}^{*}\left(\alpha, 0, \tau_{M}^{*}\left(\alpha_{m e d}, 0\right)\right)=0$, since DLAs are equivalent to saving when $\gamma=0$.
} 
optimal, with $\left.I_{M}^{*}(\alpha)<I_{M}^{*}\left(\alpha_{m e d}\right)<I^{o p t}\right)$, which induces them to reduce their over-saving, ${ }^{13}$ meaning that they move closer to the socially optimal allocation and end up better off at the majority voting equilibrium than at their most-preferred bundle $\left(I_{M}^{*}(\alpha), S_{M}^{*}(\alpha)\right)$.

By opposition, agents who are less myopic than the decisive voter $\left(\alpha>\alpha_{m e d}\right)$ are forced to consume even less insurance than they would wish when $\gamma=0\left(\right.$ since $I_{M}^{*}(\alpha)>I_{M}^{*}\left(\alpha_{m e d}\right)$ ), and they react to this lower insurance level by saving more, resulting in more over-saving and more under-insurance (compared to the optimal choice of a rational individual) than at $\left(I_{M}^{*}(\alpha), S_{M}^{*}(\alpha)\right)$, so that $W_{M}^{V}(\alpha, 0)<W_{M}^{V}(\alpha, \gamma)$ for $\gamma>0$.

We summarize the main results obtained in this section in the following proposition.

Proposition 1 When agents are myopic,

(1) they save too much and insure too little at their most-preferred allocation, because they over-estimate the returns from (private and social) insurance. Their most-preferred allocation depends on their degree of myopia (as $\alpha$ increases, saving decreases while insurance increases), but not on whether private insurance is available or not.

(2) The majority-chosen amount of social insurance is $I_{M}^{*}\left(\alpha_{m e d}\right)=\alpha_{m e d} L$ if private insurance is unavailable and nil otherwise.

(3) Agents who are more (resp., less) myopic than the median are better off (resp., worse off), at the majority-voting equilibrium, when private insurance is unavailable $(\gamma=0)$.

\section{The optimists}

Optimists know the correct average dependency probability in the economy $(\bar{\pi}=\pi)$ but underestimate their own risk both when voting and when buying DLAs. Their (perceived) indirect utility at that stage is given by

$$
\begin{aligned}
V_{O}(\alpha, \gamma, \tau)= & u\left(w(1-\tau)-s_{O}^{*}(\alpha, \gamma, \tau)-a_{O}^{*}(\alpha, \gamma, \tau)\right) \\
& +(1-\alpha \pi) u\left(R^{h}(\gamma) a_{O}^{*}(\alpha, \gamma, \tau)+s_{O}^{*}(\alpha, \gamma, \tau)\right) \\
& +\alpha \pi v\left(b(\pi)+s_{O}^{*}(\alpha, \gamma, \tau)+R^{d}(\gamma, \pi) a_{O}^{*}(\alpha, \gamma, \tau)\right),
\end{aligned}
$$

where $s_{O}^{*}(\alpha, \gamma, \tau)=s^{*}(\alpha, \gamma, \tau, \pi)$ and $a_{O}^{*}(\alpha, \gamma, \tau)=a^{*}(\alpha, \gamma, \tau, \pi)$. Unlike myopics, they make no mistake when they anticipate the returns from (social and private) insurance and their con-

\footnotetext{
${ }^{13}$ Recall from section 4 that savings and social insurance are substitutes for all agents when $\gamma=0$, so that $d s_{M}^{*}(\alpha, 0, \tau) / d \tau<0$.
} 
sumption level when dependent.

Differentiating $V_{O}(\alpha, \gamma, \tau)$ with respect to $\tau$ while making use of the envelope theorem for the optimal choices of saving and DLA, we obtain the FOC

$$
\frac{\partial V_{O}(\alpha, \gamma, \tau)}{\partial \tau}=w\left[\alpha v^{\prime}\left(d^{d}\right)-u^{\prime}(c)\right] \leq 0,
$$

for any level of $\gamma$. Comparing this equation with the FOC for $\tau$ of the myopics (equation (11)), we immediately see that optimists put a lower weight on the marginal utility when dependent. It is then clear that they under-weight this state of the world not simply because they underestimate their own risk (a feature they share with type $M$ agents), but rather because of the discrepancy between what they consider to be their own risk, and the average risk determining the LTC insurance return. In other words, it is because they consider (public and private) LTC insurance to be actuarially unfair to them that they end up putting a lower weight on the marginal benefit of this transfer. At the same time, optimists do not over-estimate LTC transfers, as myopics do.

After manipulations of the FOCs, we obtain the following ranking of marginal utilities (for any $\gamma$ and any $\alpha>0)$

$$
v^{\prime}\left(d^{d}\right)>u^{\prime}(c)>u^{\prime}\left(d^{h}\right)
$$

meaning that optimists most-prefer too little consumption when dependent and too much if autonomous (compared to the social optimum).

As in the previous section, it is helpful to reformulate the optimists preferences in terms of total saving $S$ and insurance $I$ amounts:

$$
u(w-S-\pi I)+(1-\alpha \pi) u(S)+\alpha \pi v(S+I) .
$$

We obtain from (17) that optimists save too much (a necessary condition for $d^{h}$ to be too large) at their most-preferred allocation,

$$
S_{O}^{*}(\alpha)>S^{o p t}
$$

and that they prefer too little insurance (since $d^{d}$ is too low),

$$
I_{O}^{*}(\alpha)<I^{o p t}
$$

The intuition for this result runs as follows: optimists want too little insurance, because their optimism induces them both to put too low a weight on the dependency probability, and to 
consider insurance (whether public or private) as actuarially unfair to them. Buying too little insurance decreases the marginal cost of saving while increasing its marginal benefit, increasing their most-preferred saving (which is not affected by any apparent actuarial unfairness) amount above $S^{\text {opt }}$.

Comparing with the most-preferred allocation of type $\mathrm{M}$ agents, we see that the main difference is that myopics equalize marginal utilities when autonomous and when young, while optimists have a larger marginal utility when young. Both types of agents end up with too little consumption when dependent. It is not possible to infer from these relationships a comparison of the most-preferred saving and insurance amounts of the two types of agents. ${ }^{14}$ Indeed, we obtain using numerical simulations ${ }^{15}$ that these comparisons can go both ways depending on the parameters used.

Manipulations of (8), (9) and (16) show that $I_{O}^{*}(\alpha)$ is zero below some threshold value of $\alpha$, and then increasing in $\alpha$. In contrast to the myopics, optimists with a very large behavioral bias (i.e., a very low value of $\alpha$ ) prefer no insurance at all because insurance looks too unfair to them. Denoting by $\tau_{O}^{*}(\alpha, \gamma)$ the most-preferred income tax rate of type O agents, we obtain that, for values of $\alpha$ low enough that $\tau_{O}^{*}(\alpha, \gamma)=a_{O}^{*}\left(\alpha, \gamma, \tau_{O}^{*}(\alpha, \gamma)\right)=I_{O}^{*}(\alpha)=0$, the optimal amount of saving $s_{O}^{*}\left(\alpha, \gamma, \tau_{O}^{*}(\alpha, \gamma)\right)>0$ satisfies

$$
u^{\prime}(w-s)=(1-\alpha \pi) u^{\prime}(s)+\alpha \pi v^{\prime}(s),
$$

(since $u^{\prime}(0)=\infty$ ) and is increasing in $\alpha$. As agents become less optimistic (i.e., as $\alpha$ increases), they put more weight on the high marginal utility state of the world (dependency) and thus save more. Observe that these results hold whether $\gamma$ is nil or positive, and that the threshold value of $\alpha$ below which $I_{O}^{*}(\alpha)=0$ is not affected by $\gamma$ either.

Whatever the value of $\gamma$, application of the implicit function theorem on (8), (9) and (16) when $\alpha$ is large enough that $I_{O}^{*}(\alpha)>0$ shows, as for myopics, that as $\alpha$ increases optimists substitute more insurance to less saving : $d S_{O}^{*}(\alpha) / d \alpha<0$ and $d I_{O}^{*}(\alpha) / d \alpha>0$. As $\alpha$ increases, optimists put more weight on the dependency state and consider insurance to be less actuarially unfair. This increases their demand for total insurance, $I_{O}^{*}$. On the one hand, a larger demand for insurance increases the marginal cost of saving (since it increases the marginal utility of first-period consumption) while decreasing its benefit (since marginal utility when dependent

\footnotetext{
${ }^{14}$ Unlike for myopics, obtaining explicit formulations for the most-preferred saving and insurance amounts of optimists would require to specify a functional form for the utility function.

${ }^{15}$ They are available upon request to the authors.
} 
decreases). This in turn decreases the individual's demand for private savings. On the other hand, the fact that a less optimistic individual puts more weight on the dependency state leads mechanically to a larger marginal utility from saving, since $v^{\prime}\left(d^{d}\right)>u^{\prime}\left(d^{h}\right)$. This latter effect is smaller than the former, leading less optimistic individuals to prefer less saving $S_{O}^{*}(\alpha)$ as $\alpha$ increases.

Observe then that, unlike for myopics, optimal saving is not monotone in the degree of behavioral bias, since saving first increases with $\alpha$ (when $\alpha$ is low enough that agents do not want to insure themselves) and then decreases with $\alpha$ (when $\alpha$ is large enough that agents substitute more insurance to less saving).

As for the voting equilibrium, we obtain a result similar to the case of myopic agents. When private insurance is available $(\gamma>0)$, social and private insurance are substitutes, and the majority-chosen value of $\tau, \tau_{O}^{V}(\gamma)$, is zero: agents rather buy their individually most-preferred amount of private insurance than being forced to consume too much (social) insurance. When private insurance is not available $(\gamma=0)$, the most-preferred value of $\tau$ is (weakly) increasing with $\alpha$, and with single-peaked (indeed concave) preferences, the majority-chosen value of $\tau$ is the one most-preferred by the agent with the median ability level: $\tau_{O}^{V}(0)=\tau_{O}^{*}\left(\alpha_{\text {med }}, 0\right)$.

We can replicate the argument made for myopics to show that agents who are more (resp., less) optimistic than the median are better off (resp., worse off), at the majority-voting equilibrium, when private insurance is unavailable. Formally, we denote by $W_{O}^{V}(\alpha, \gamma)$ the real utility attained by optimists at the majority-voting equilibrium, defined as ${ }^{16}$

$$
\begin{aligned}
& W_{O}^{V}(\alpha, \gamma)=W_{O}^{*}\left(I_{O}^{*}(\alpha), S_{O}^{*}(\alpha)\right) \text { if } \gamma>0, \\
& W_{O}^{V}(\alpha, 0)=W_{O}^{*}\left(w \tau_{O}^{*}\left(\alpha_{m e d}, 0\right) / \pi, s_{O}^{*}\left(\alpha, 0, \tau_{O}^{*}\left(\alpha_{\text {med }}, 0\right)\right)\right) \text { if } \gamma=0 .
\end{aligned}
$$

We have that $W_{O}^{V}(\alpha, 0)>W_{O}^{V}(\alpha, \gamma)$ when $\gamma>0$ for agents with $\alpha<\alpha_{m e d}$ if $\tau_{O}^{*}\left(\alpha_{m e d}, 0\right)>0$, while the opposite holds for individuals with $\alpha>\alpha_{m e d}$.

In a nutshell, when private insurance is available, no social insurance is publicly provided and agents have the same utility under majority voting as at their most-preferred allocation. When private insurance is not available, everybody is forced to consume the amount of insurance most-preferred by the agent with the median degree of optimism. Very optimistic individuals then benefit from being forced to consume more insurance than they would wish, while being

\footnotetext{
${ }^{16}$ As for type M, we assume w.l.o.g. that $a_{O}^{*}=0$, since DLAs are equivalent to saving when $\gamma=0$.
} 
induced to reduce their over-saving. Individuals who are less optimistic than the median suffer from being obliged to consume even less insurance than the amount they most-prefer, and react by further increasing their already too large saving amount. The only caveat is that this result holds only if $\tau_{O}^{*}\left(\alpha_{m e d}, 0\right)>0$ : if $\tau_{O}^{*}\left(\alpha_{m e d}, 0\right)=0$, then all individuals more optimistic than the median also prefer no insurance and thus obtain the same utility at the majority-voting equilibrium whatever the value of $\gamma: W_{O}^{V}(\alpha, 0)=W_{O}^{*}\left(0, S_{O}^{*}(\alpha)\right)=W_{O}^{V}(\alpha, \gamma), \forall \gamma$ and $\alpha \leq \alpha_{m e d}$.

We summarize the main results of this section in the following proposition.

Proposition 2 When agents are optimists,

(1) they save too much and insure too little at their most-preferred allocation, because they consider (private and social) insurance to be actuarially unfair to them. Unlike myopics, very biased optimists prefer no insurance at all. Their most-preferred allocation depends on their degree of myopia (as $\alpha$ increases, saving first increases and then decreases, while insurance weakly increases), but not on whether private insurance is available or not.

(2) The majority-chosen amount of social insurance is the most-preferred amount of the individual with the median behavioral bias, $\alpha_{m e d}$, if private insurance is unavailable and nil otherwise. (3) Agents who are more (resp., less) myopic than the median are better off (resp., worse off), at the majority-voting equilibrium, when private insurance is unavailable.

\section{The sophisticated procrastinators}

In this section, we assume that, at the time agents vote, they are all sophisticated, anticipating that their future choice of LTC annuity and of savings will be made according to their degree of procrastination $\alpha$. Recall also that procrastinators know the correct average prevalence of dependency in the economy, so that $\bar{\pi}=\pi$. Type $\mathrm{S}$ individual's (real and perceived) indirect utility when voting is then

$$
\begin{aligned}
V_{S}(\alpha, \gamma, \tau)= & u\left(w(1-\tau)-s_{S}^{*}(\alpha, \gamma, \tau)-a_{S}^{*}(\alpha, \gamma, \tau)\right) \\
& +(1-\pi) u\left(R^{h}(\gamma) a_{S}^{*}(\alpha, \gamma, \tau)+s_{S}^{*}(\alpha, \gamma, \tau)\right) \\
& +\pi v\left(b(\pi)+s_{S}^{*}(\alpha, \gamma, \tau)+R^{d}(\gamma, \pi) a_{S}^{*}(\alpha, \gamma, \tau)\right),
\end{aligned}
$$

where $s_{S}^{*}(\alpha, \gamma, \tau)=s^{*}(\alpha, \gamma, \tau, \pi)$ and $a_{S}^{*}(\alpha, \gamma, \tau)=a^{*}(\alpha, \gamma, \tau, \pi)$.

We obtain the following proposition. 
Proposition 3 (1) $\forall 0 \leq \gamma \leq 1$, S agents unanimously most-prefer the insurance and saving amounts that correspond to the first-best allocation :

$$
u^{\prime}(c)=u^{\prime}\left(d^{h}\right)=v^{\prime}\left(d^{d}\right)
$$

(2) $\forall 0 \leq \gamma \leq 1$, S agents unanimously vote for the social insurance rate that decentralizes the first-best allocation, and no one buys any private insurance/DLA. S agents then attain the first-best utility level at the majority-voting equilibrium, whether private insurance is available or not.

Proof. See appendix.

The intuition for this proposition runs as follows. By setting the tax rate such that the social insurance level is optimal (i.e., such that $b(\tau)=L$ ), S agents equalize their marginal utility when autonomous and when dependent. This in turn neutralizes the impact of procrastination on the saving choice, because it becomes immaterial that $\mathrm{S}$ agents under-weight their utility if dependent when saving. Agents then have no wish to buy any private insurance. This results into a unanimous support for the first-best amount of social insurance coupled with no one complementing on the private market.

\section{Conclusion and extensions}

Our model confirms the intuition that behavioral biases may lead to underprovision of (social or private) LTC insurance, except for the sophisticated procrastinators. Myopics and optimists favor too little LTC insurance, but for different reasons and with a different and testable implication: unlike myopics, very biased optimists have no demand at all for even an actuarially fair LTC insurance (whether public or private). In our model, private insurance is available as part of a DLA, and we obtain that the fraction of DLA that is devoted to private insurance has no impact on how much total saving and insurance biased agents most-prefer. The availability of private insurance is crucial for both myopics and optimists at the majority voting equilibrium, as we show that availability of private insurance is beneficial to agents less biased than the median, but at the expense of those whore are more biased than the median.

We have seen in footnote 4 in the Introduction that the empirical evidence on behavioral biases points to the possibility that some individuals actually over-estimate their dependency risk (i.e., have $\alpha>1$ ). Although the analytical results we obtain with sophisticated procrastinators 
carry through to the case where some (or even all) exhibit $\alpha>1$, it seems to us difficult to rationalize the over-estimation of the dependency risk as some type of procrastination. This over-estimation can still be explained as "myopia" or, in this case, pessimism, and our analytical results also carry through for these two types of agents. More precisely, type $\mathrm{M}$ and type $\mathrm{O}$ agents with $\alpha>1$ prefer too much LTC insurance and too little saving, but as long as the median bias is lower than one, the majority voting results are not affected.

Our results can easily be extended to the case where the three types of agents coexist in society (while keeping the continuous distribution of biases for all types). The key insight is that individual preferences for (social or private) LTC insurance do not depend on the composition of society. Type S individuals still prefer the socially optimal amount of social (and total) insurance. When private insurance is available, all type $\mathrm{O}$ and type $\mathrm{M}$ agents still prefer no social insurance at all, which remains the majority chosen equilibrium (unless type $\mathrm{S}$ voters constitute a majority). When private insurance is not available, all type $\mathrm{O}$ and type $\mathrm{M}$ agents prefer a lower-than-optimal amount of social insurance, so that the majority chosen level of social insurance remains sub-optimal. Finally, type $\mathrm{O}$ and type $\mathrm{M}$ agents who most-prefer less insurance than the majority-chosen level (because they are more biased than the decisive voter) still end up being better off (at the majority voting equilibrium) than if private insurance were available, while the opposite applies to those who most-prefer more insurance.

Our framework is admittedly very simple, with agents identical in income, LTC risk and lifetime expectancy and differing only in bias type and intensity. Having different income and risk levels would break the perfect substitutability between private and social LTC insurance, with for instance, social insurance redistributing between income and, ex ante, risk levels while private insurance remains actuarially fair, as in De Donder and Pestieau (2011). Also, introducing heterogeneity in lifetime expectancies would make disability linked annuities more appealing to certain individuals, and would allow to study the consequence of the correlation between lifetime expectancy and dependency risk. We leave these extensions to future research.

\section{Appendix}

\section{Proof of Proposition 3}

The preferred tax rate of a type $\mathrm{S}$ individual is denoted by $\tau_{S}^{*}(\alpha, \gamma)$ and, if positive, is such 
that

$$
\begin{aligned}
\frac{\partial V_{S}(\alpha, \gamma, \tau)}{\partial \tau}= & w\left[v^{\prime}\left(d^{d}\right)-u^{\prime}(c)\right] \\
& +\frac{d a_{S}^{*}(\alpha, \gamma, \tau)}{d \tau}\left[-u^{\prime}(c)+(1-\pi) R^{h} u^{\prime}\left(d^{h}\right)+\pi R^{d} v^{\prime}\left(d^{d}\right)\right] \\
+\quad & \frac{d s_{S}^{*}(\alpha, \gamma, \tau)}{d \tau}\left[-u^{\prime}(c)+(1-\pi) u^{\prime}\left(d^{h}\right)+\pi v^{\prime}\left(d^{d}\right)\right]=0 .
\end{aligned}
$$

Assuming that the most-preferred saving amount is positive when $\tau=\tau_{S}^{*}(\alpha, \gamma)$, the first-order condition for saving (8) holds with equality and we can rewrite the above expression as

$$
\begin{aligned}
\frac{\partial V_{S}(\alpha, \gamma, \tau)}{\partial \tau} & =\left[v^{\prime}\left(d^{d}\right)-u^{\prime}\left(d^{h}\right)\right]\left((1-\alpha \pi) w+\left(\frac{d a_{S}^{*}(\alpha, \gamma, \tau)}{d \tau}+\frac{d s_{S}^{*}(\alpha, \gamma, \tau)}{d \tau}\right)(\pi(1-\alpha)+\gamma(1-\pi))\right) \\
& =0
\end{aligned}
$$

so that $\tau_{S}^{*}(\alpha, \gamma)$ is such that $v^{\prime}\left(d^{d}\right)=u^{\prime}\left(d^{h}\right)$. Plugging this equality into (8), we obtain that

$$
v^{\prime}\left(d^{d}\right)=u^{\prime}(c)=u^{\prime}\left(d^{h}\right) .
$$

If $\gamma>0$, we then obtain that the FOC for $a,(9)$ is negative for all $a$ so that $a_{S}^{*}\left(\alpha, \gamma, \tau_{S}^{*}(\alpha, \gamma)\right)=0$. This in turn guarantees that $s_{S}^{*}\left(\alpha, \gamma, \tau_{S}^{*}(\alpha, \gamma)\right)>0$, since we have assumed that $u^{\prime}(0) \rightarrow+\infty$. If $\gamma=0, a$ is a perfect substitute to $s$ (equations (8) and (9) are equivalent), so that we can assume w.l.o.g. that $a_{S}^{*}\left(\alpha, \gamma, \tau_{S}^{*}(\alpha, \gamma)\right)=0$ which in turn guarantees that $s_{S}^{*}\left(\alpha, \gamma, \tau_{S}^{*}(\alpha, \gamma)\right)>0$.

\section{References}

[1] Bacon, P. W., L. J. Gitman, K. Ahmad, and M. F. Ainina, 1989, Long-Term Catastrophic Care: A Financial Planning Perspective, Journal of Risk and Insurance, 56(1): 146-154.

[2] Brown, J.R. and A. Finkelstein, 2007, Why is the Market for Long-Term Care Insurance so Small?, Journal of Public Economics, 2007, 1967-1991.

[3] Brown, J.R. and A. Finkelstein, 2008, The Interaction of Public and Private Insurance: Medicaid and the Long-Term Care Insurance Market, American Economic Review, 98, $1083-1102$.

[4] Brown, J.R. and A. Finkelstein, 2009, The Private Market for Long-Term Care Insurance in the United States: a Review of the Evidence, Journal of Risk and Insurance, 76, 5-29. 
[5] Brown, J.R. and A. Finkelstein, 2011, Insuring Long-Term Care in the United States, Journal of Economic Perspectives, 25(4), 119-142.

[6] Cremer H., P. De Donder, D. Maldonado and P. Pestieau, 2007, Voting over the type and generosity of a pension system when some individuals are myopic, Journal of Public Economics, 91, 2041-2061.

[7] Cremer H., P. De Donder and P. Pestieau, 2009, Providing sustainable long term care: A looming challenge, TSE Note $n^{\circ} 3$. http://www.tsefr.eu/images/TSE/TSENotes/tsenotes3.pdf

[8] Cremer H. and K. Roeder, 2012, Long-term care policy, myopia and redistribution. TSE WP 12-314.

[9] De Donder P. and P. Pestieau, 2011, Private, social and self insurance for long-term care: A political economy analysis. CORE DP 53.

[10] Finkelstein, A. and K. McGarry, 2006, Multiple Dimensions of Private Information: Evidence from the Long-Term Care Insurance Market, The American Economic Review, 96, 938-958.

[11] Finkelstein, A., E. Luttmer and M. Notowidigdo, 2013, What good is wealth without health? The effect of health on the marginal utility of consumption, Journal of the European Economic Association 11, 221-258.

[12] Genworth, 2012, Cost of Care Survey. Available at http://www.genworth.com/content/non_navigable/corporate/about_enworth/industry_expertise/cost_of_care.html

[13] Haavio M. and K. Kotakorpi, 2011, The political economy of sin taxes, European Economic Review 55 (4), 575-594.

[14] Hamermesh, D., 1985, Expectations, life expectancy and economic behaviour. Quarterly Journal of Economics 100 (2), 389-408

[15] Levantesi S. and M. Menzietti, 2012, Longevity and disability risk analysis in enhanced life annuities, Insurance: Mathematics and Economics 50: 391-401 
[16] Ludwig, A., Zimper, A., 2007, A Parsimonious Model of Subjective Life Expectancy. MEA, University of Manheim, pp. 2007-2065

[17] Mayhew L., Karlsson M. and Rickayzen B, 2010, The role of private finance in paying for long-term care, The Economic Journal, 120, 478-504.

[18] Mossin J., 1968, Aspects of rational insurance purchasing, Journal of Political Economy, 76 , p. $533-568$.

[19] Nuscheler R. and K. Roeder, 2010, The Political Economy of Long-Term Care, mimeo.

[20] O'Donoghue, T. and M. Rabin (2003), Studying optimal paternalism, illustrated by a model of sin taxes, American Economic Review (Papers and Proceedings) 93, 186-191.

[21] OECD, 2011, Help Wanted? Providing and Paying for Long-Term Care, available at http://www.oecd.org/document/23/0,3746,en_2649_37407_47659479_1_1_1_37407,00.html

[22] Pauly, M. V., 1990, The Rational Nonpurchase of Long-Term-Care Insurance, The Journal of Political Economy, 98: 153-168.

[23] Pestieau, P. and G. Ponthière, 2010, Long-Term Care Insurance Puzzle, CORE DP 2010/23.

[24] Rickayzen B, 2007, An Analysis of Disability-linked Annuities, CASS business school, Actuarial Research Paper 180.

[25] Salanie, F. and N. Treich, 2009, Regulation in Happyville, The Economic Journal 119, 665-679.

[26] Sjoblom K., 1985, Voting for Social Security. Public Choice 45 (3), 225-240

[27] Sloan, F.A. and E.C. Norton, 1997, Adverse Selection, Bequests, Crowding Out, and Private Demand for Insurance: Evidence from the Long-Term Care Insurance Market, Journal of Risk and Uncertainty, 15, 201-219.

[28] Thaler, R. and C. Sunstein (2003), Behavioral economics, public policy, and paternalism, American Economic Review (Papers and Proceedings) 93, 175-179.

[29] Zhou-Richter, T., M. Browne, and H. Gründl, 2010, Don't They Care? Or, Are They Just Unaware? Risk Perceptions and the Demand for Long-Term Care Insurance, Journal of Risk and Insurance, 77 (4), 715-747. 